\title{
Planar free-standing metal layer fabrication: implementing sub-structures in micromirror arrays for light steering applications
}

\author{
Natalie Worapattrakul ${ }^{1,2^{*}}$ (D) Andreas Tatzel ${ }^{1,2}$, Volker Viereck ${ }^{1,2}$ and Hartmut Hillmer ${ }^{1,2}$
}

\begin{abstract}
We present a method to fabricate planar metal layers to be used as micromachined mirrors. Released mirrors of pure metal involve severe stress and reveal specific challenges to obtain planar mirror structures. Introducing sub-structures generating corrugated patterns, the metal mirror layers can be mechanically stabilized and undesired mirror bending can be reduced. For our investigations we used different arrangements of line structures on our metal mirrors, such as a group of straight or curved lines oriented differently. Comparing all the implemented different designs, planar micromirrors were achieved via sub-structures with a combination of straight lines arranged orthogonally to a single line. These planar micromirrors allow steering of the incident light by reflection and adjustment of the window transmittance. The presented low-cost method is suitable for large area fabrication of micromirror arrays, but also can be customized for other applications, where planar free-standing metal layers are required.
\end{abstract}

Keywords: Electrostatic actuation, Energy saving, Fabrication technologies, Light steering, MEMS/MOEMS, Micromirror arrays, Stress compensation

\section{Introduction}

Advantages in terms of high reliability, low power consumption, minimum weight and size, high stability, lowcost, and large area fabrication have promoted huge interest in the field of microelectromechanical systems (MEMS) and advanced applications of these systems in various sectors $[1,2]$. However, the aspect of energy efficiency and energy saving by applying these systems has not been sufficiently exploited. We devote our research to this goal by focusing on improving energy efficiency of buildings. By using daylight for indoor lighting/heating or blocking daylight to prevent rooms from heating-up, we expect a drastic reduction of the load on cooling and heating units and the need of artificial light. Especially

\footnotetext{
*Correspondence: worapattrakul@gmail.com

${ }^{1}$ Institute of Nanostructure Technologies and Analytics (INA), Faculty

of Electrical Engineering and Computer Science, Department

of Technological Electronics, University of Kassel, Heinrich-Plett-Strasse 40,

34132 Kassel, Germany

Full list of author information is available at the end of the article
}

the use of daylight for illumination in buildings is challenging: in summer the sun rises high so that areas near the windows are bright, but the depth of the room stays dark; in winter the low position of the sun causes glare. To meet this challenge electrostatically actuated micromirrors were chosen for daylight steering purposes applied between window panes [3-5]. Since these MEMS structures are applied to manipulate light, they can also be classified as micro-optoelectromechanical systems (MOEMS). The mirrors with dimensions of a few $100 \mu \mathrm{m}$ are made of thin stressed metal layers, which in the normal case results in a curved structure, as is known from microshutters [6], shown in Fig. 1a. In this case the structures can be selectively opened (full daylight transmittance) or closed (complete reflection) by electrostatic actuation to modulate the incident light through a transparent substrate. The goal of our research group, however, is to fabricate micromirrors, which also allow steering of the incident light via reflection and adjustment of the window substrate transmittance. This can only be achieved with metal mirrors that contain planar 


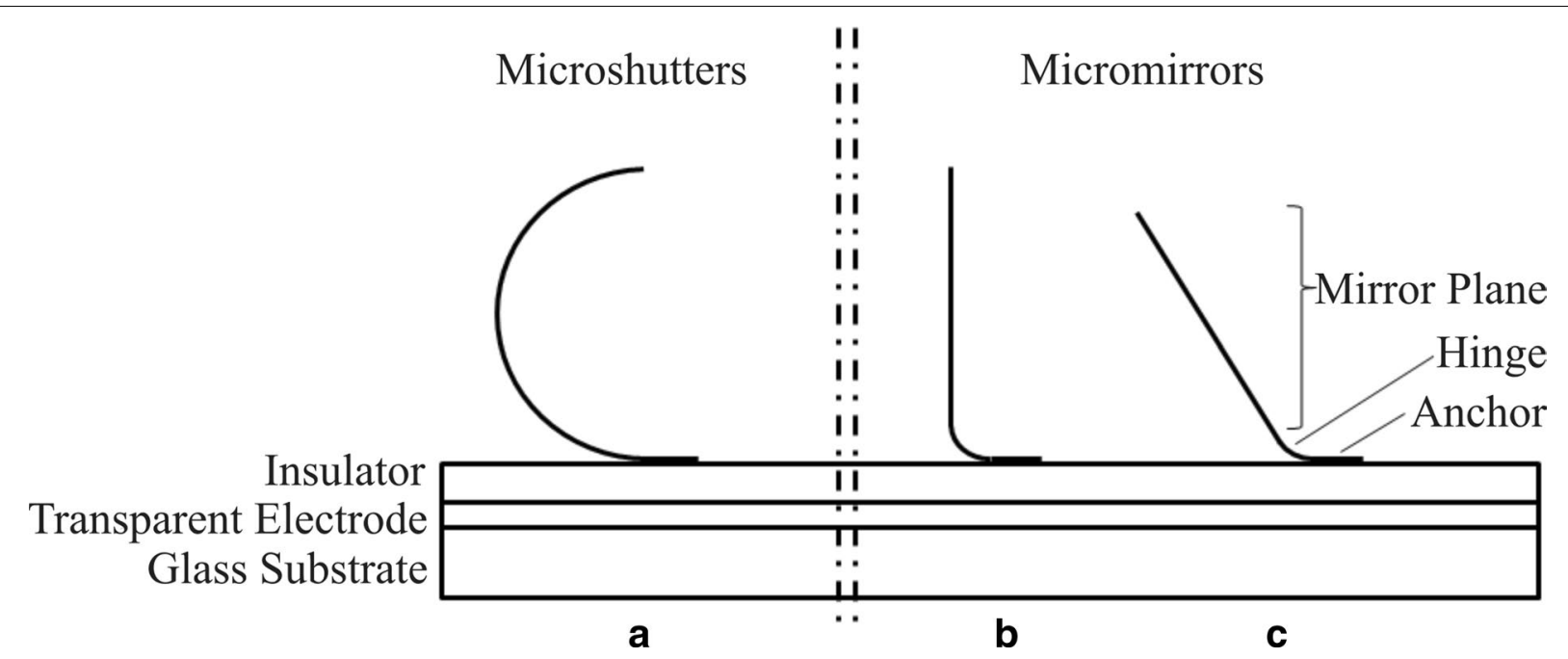

Fig. 1 Cross section of microshutters in opened state (a), micromirrors in opened non-actuated state (b), and in an intermediate state during actuation (c)

reflecting areas. Thus, our micromirror structures can be divided into 3 areas as shown in Fig. 1b, c:

1. The anchor area, which allows the attachment of the mirrors to the substrate.

2. The curved hinge area, which is obtained by introducing intrinsic stress in the metal layer through specific deposition parameters [7], which leads to opened mirrors in the non-actuated state. The mirrors presented in this paper consist of an aluminum (Al) bi-layer deposited by physical vapor deposition (PVD), applying an electron beam source for the first layer of $200 \mathrm{~nm} \mathrm{Al}$ followed by a further $200 \mathrm{~nm} \mathrm{Al}$ layer deposition from a resistance heated evaporation source. According to literature these single layers, deposited with PVD under comparable conditions, should give rise to nearly stress free-layers [8], however once combining these 2 layers the stress of the new layer system increases noticeably. The investigation of the cause for this intrinsic stress was not included in the scope of this research, but was performed and described in detail in the EU research project MEM4WIN [9]. In this project such an $\mathrm{Al}$ bi-layer was examined by transmission electron microscopy (TEM) and Auger depth profile analysis, revealing an interface layer between the 2 deposited $\mathrm{Al}$ layers, which shows a high level of oxygen. Thus, it was assumed that the stress in the $\mathrm{Al}$ bi-layer originates from $\mathrm{Al}_{2} \mathrm{O}_{3}$, which was formed by the reaction of $\mathrm{Al}$ and Oxygen in this area. Since the stress value for $\mathrm{Al}_{2} \mathrm{O}_{3}$ of $300-350 \mathrm{MPa}$ from literature [10] is in the same range as the stress we observed for the applied Al bi-layer in this research, this hypothesis is further substantiated.

3. The planar mirror plane, which is the main part to interact with the incident light.

To enable the electrostatic actuation of these micromirrors further components, namely an insulator layer and a transparent electrode on a glass substrate are required (the same applies to microshutters). The main concept, the technological fabrication and various experimental results are presented in detailed papers [11, 12].

In order to obtain planar mirror areas, local planarization is required. For compound semiconductors, dielectrics or in this case stressed metal layers, the planarization is commonly accomplished by introducing an additional tailored counter-stressed layer on the corresponding area [13]. Alternatively the planarization can also be achieved by adding a nearly stress-free layer with larger thickness compared to the compensated layer. Note that this additional effort is not required for $\mathrm{Si}$ based MEMS, where flat mirror are easily obtained [14]. These approaches have in common that additional layers have to be deposited and structured to define the planarized area. This results in a more sophisticated fabrication procedure with further production costs and a complex layer system, which may negatively influence the device function. To avoid these drawbacks we propose a new method to define planar areas in stressed metal layers without requiring additional compensation layers. Introducing sub-structures we generate corrugated patterns, which mechanically stabilize these metal mirror layers and efficiently reduce undesired mirror bending. This 
idea derives from observations of corrugated patterns on the macroscopic scale (for e.g. corrugated metal sheets applied in the construction sector), where the stiffness along the corrugation is perceivably increased, motivating us to find the optimum sub-structure design providing a planar metal layer in the structured area.

\section{Sub-structure design and method of fabrication}

In order to find the most suitable sub-structure design, which results in planarization of the structured area, different arrangements were investigated. 3 sub-structure designs (I-III) presented in this letter are shown in Fig. 2. The idea of each design is based on the aforesaid stabilization through corrugated structures. In design I this was pursued by applying concentric circles originating from the corners opposite to the anchor of the mirrors. Design II consists of parallel lines along the short sides of the mirrors (along $y$-axis) to prevent bending of the mirrors around the $\mathrm{x}$-axis. Design III introduces an additional line along the long side of the mirrors (along $\mathrm{x}$-axis) to further stabilize and prevent bending of these mirrors around the $y$-axis.

Micromirrors with these chosen sub-structure designs were fabricated by a process based on photolithography. $3 \mathrm{D}$ sacrificial structures made of photoresist $A Z^{\circledR} \mathrm{nLOF}$ 2070 (Microchemicals $\mathrm{GmbH}$ ) are fabricated on a glass substrate (Fig. 3a) using 3 photoresist layers, which are structured consecutively above one another. This generates the 3 components: mirror area, sub-structures, and separating walls. After each step the photoresist is hard baked to maintain its shape. These 3D sacrificial structures act as shaping structures in the following deposition step of the intrinsically stressed aluminum layer (Fig. 3b). Since an anisotropic deposition method was applied, sidewall deposition in the separating walls is avoided. The solvent TechniStrip NI555 (Microchemicals GmbH) is used to selectively remove the sacrificial structures underneath the metal layer. This enables the lift-off of the separating walls and mirror release. Finally the mirrors only remain attached to the glass substrate by the anchor area, establishing the desired opened state (Fig. 3c).

Even though the implementation of sub-structures requires an additional step in the fabrication process, it is only a single conventional photolithography step. This is relatively simple, in comparison to the planarization by a compensation layer, where at least 2 further steps: a stress-tailored material deposition and structuring of this material to define the planarized area are required [13].

An even more valuable advantage of the presented fabrication concept can be exploited, when transferring the fabrication to a process based on nanoimprint technology $[15,16]$, where 3D structures can be shaped
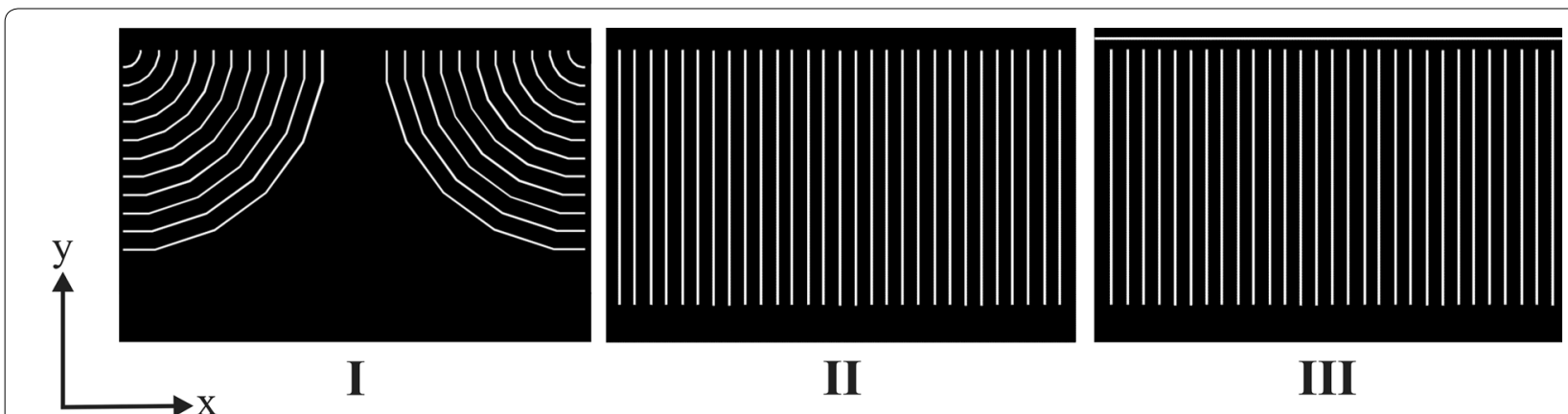

Fig. 2 Sub-structure designs I-III with defined $x$ - and $y$-axis. The bottom edges of the designs present the hinge area, connected to the anchor, respectively
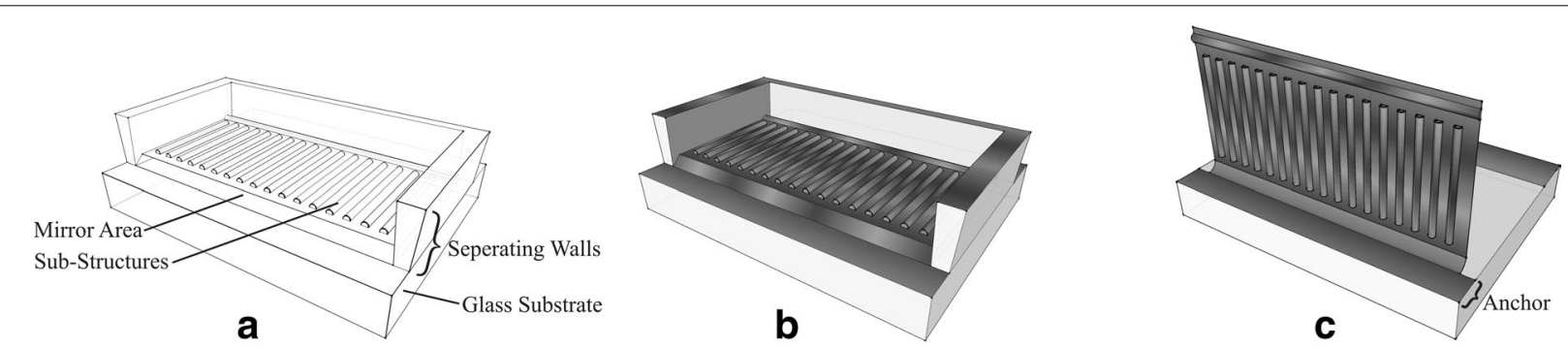

Fig. 3 A representative 3D sacrificial structure (with sub-structures design III) made of photoresist (a), after deposition of stressed aluminum layer depicted in grey (b), and released micromirror in non-actuated state (c). Insulator and transparent electrode are not shown here 
in a single process step. Since this technology facilitates a rapid, low-cost, and large area fabrication, it is highly suitable for industrial production, which is the final aim of our research work on micromirrors in light steering applications.

\section{Experimental results and discussion}

Micromirrors including 3 sub-structure designs (Fig. 2) were successfully fabricated. The influence of these sub-structures on the planarity behavior of the mirror area is focused in our study. The profiles of the mirrors in opened state were investigated by means of scanning electron microscopy (SEM), whereby the planarity of the micromirrors were determined by examining the crosssection of these mirrors. In general, we observed that the implementation of sub-structures into stressed metal layers has a strong impact on the behavior of these metal mirror layers, resulting in profiles significantly different from the curved structures of microshutters [6]. Micromirrors containing sub-structures with design I exhibited mirror curling expanding out from the edges, preventing them to rise up from the substrate (Fig. 4a). Micromirrors with design II sub-structures, on the other hand, met the expectations by successfully suppressing bending of the mirrors around the $x$-axis. Bending around the $y$-axis, however, was still present in form of curling originating from the short sides of the mirrors, resulting in non-planar micromirrors (Fig. 4b).

Our goal to fully planarize the structured mirror area was achieved by sub-structures with design III (Fig. 5a), where a combination of lines along the $\mathrm{x}$ - and $\mathrm{y}$-axis of the mirrors suppressed bending of the metal layers in respective directions. A single additional line along the long side of the mirrors (along $\mathrm{x}$-axis) was sufficient to eliminate bending around the $y$-axis of these mirrors (compare design II, Fig. 4b), since the anchors also contribute to the stabilization of the mirrors in the same direction. The corresponding structured photoresist layer (Fig. 5b close-up) analyzed by atomic force microscopy (AFM) and the resulting micromirrors with planar mirror planes are depicted in Fig. 5c-e.

For the sub-structure design III, some alteration of the parameters including position of the long horizontal line (defined by geometric parameters $y$ and $z$ ), length of the parallel lines $x$, linewidth as full width at half maximum values (FWHM), and height of the sub-structures were also considered. Table 1 depicts the possible range for each value, which reveals planarization of the mirror plane (distance between parallel lines $v$ and hinge length $w$ kept constant at $11 \mu \mathrm{m}$ and $20 \mu \mathrm{m}$, respectively).

Besides the sub-structure design, the shape of the hinge also plays an important role for the functionality of the final micromirrors. In order to gain maximum transmission of light in the non-actuated opened state, not only the mirror plane has to be completely planarized, but also the hinge has to exhibit a suitable curvature to reveal a $90^{\circ}$ angle between the mirror plane and the surface of the substrate. For our defined hinge length $w$ the suitable curvature $r_{\mathrm{c}}$ is equal to $2 w / \pi=12.7 \mu \mathrm{m}$.

For the fabricated micromirrors in this study a maximum transmission in opened state of $85 \%$ and a minimum transmission in closed state of $5 \%$ were estimated. These values were calculated from the geometrical coverage of the opaque and transparent areas. Since the scope of this study was the planarization of the mirror plane area and not the actuation voltage or minimum and maximum transmission, we intentionally designed the mirrors with large gaps to the neighboring mirrors, in order to isolate our study from possible parasitic influences. However, other micromirror studies in our research group having optimized mirror array arrangements demonstrated a minimum transmission of $0.01 \%$
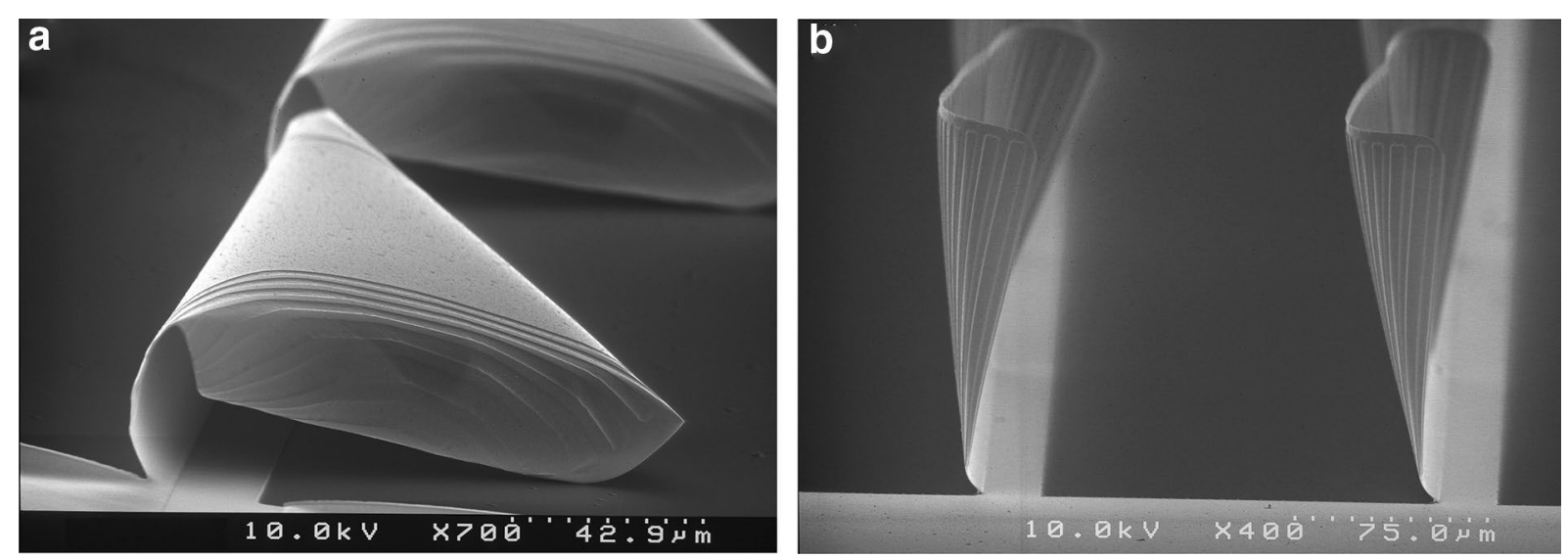

Fig. 4 SEM images of micromirrors containing sub-structures with design I (a) and design II (b) 

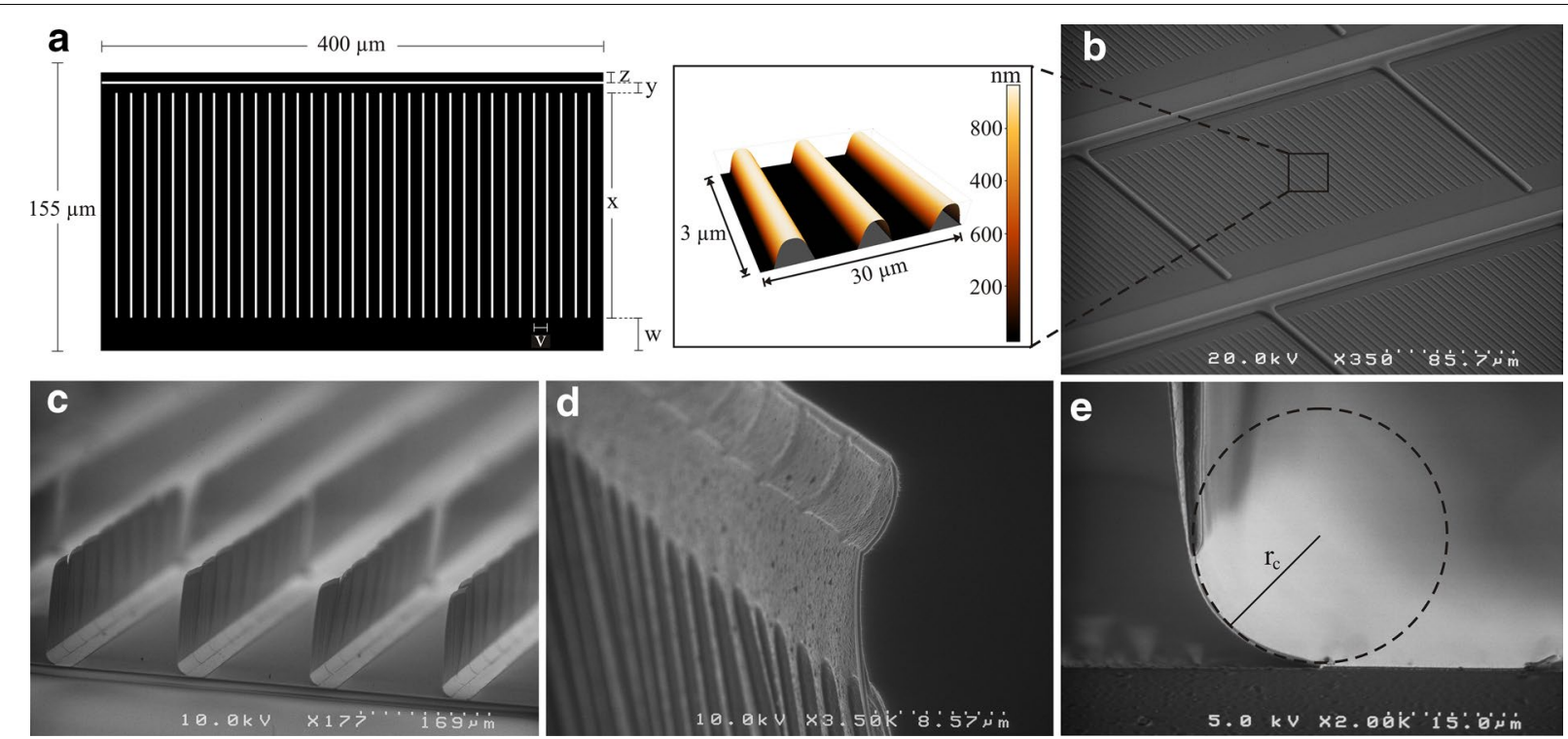

Fig. 5 Schematic of sub-structure design III with mirror dimensions and geometric parameters $v-Z(\mathbf{a})$, profile of the corresponding structured photoresist layer analyzed by SEM and a close-up of the parallel lines of design III analyzed by AFM (b), SEM image of the resulting micromirrors with entirely planar mirrors (c), close-up SEM image of the mirror plane corner (d), close-up SEM image of the hinge area with the depicted radius of curvature $r_{c}(e)$. Within our structure screening, design III was identified to work best

Table 1 Values for geometric parameters $x-z$ of substructure design III depicted in Fig. $5 a$ and height and linewidth values originating from AFM measurement in Fig. $5 \mathbf{b}$

\begin{tabular}{ll}
\hline Geometric Parameters & Possible Range of Values \\
\hline$x$ & $109.3-112.8 \mu \mathrm{m}$ \\
$y$ & $10.1-11.1 \mu \mathrm{m}$ \\
$z$ & $6.1-8.6 \mu \mathrm{m}$ \\
Height & $0.6-0.9 \mu \mathrm{m}$ \\
Linewidth (FWHM) & $3.1-4.5 \mu \mathrm{m}$ \\
\hline
\end{tabular}

and a maximum transmission of $73 \%$, revealing a contrast of 7300. The smallest voltage to fully actuate the mirrors was $12 \mathrm{~V}$, while typical actuation voltages for our micromirrors lie between 20 and $40 \mathrm{~V}[11,12]$.

\section{Conclusion}

We proposed a new method to fabricate planar metal layers without requiring additional compensation layers. The method is based on mechanical stabilization through corrugated structures in the target metal layer introduced via sub-structures. The optimum sub-structure design, which results in a planar mirror plane, consists of a combination of straight lines arranged orthogonally to a single line. Applying this method we have successfully fabricated micromirrors that can be applied to both steer the incident light and to adjust the transmittance of the substrate. However, this method can also be customized for other applications where planar free-standing metal layers are required. Our further research will focus on developing a micromirror fabrication process based on nanoimprint technology to facilitate an easy transfer to industrial production.

\section{Abbreviations \\ INA: Institute of Nanostructure Technologies and Analytics; CINSaT: Center for Interdisciplinary Nanostructure Science and Technology; MEMS: Microelectro- mechanical Systems; MOEMS: Micro-Optoelectromechanical Systems; SEM: Scanning Electron Microscopy; AFM: Atomic Force Microscopy; FWHM: Full Width at Half Maximum.}

\section{Acknowledgements}

The authors thank all the colleagues at the Institute of Nanostructure Technology and Analytics for stimulating discussions and technological support. Financial support by the Doctoral Scholarship Program of the German Federal Environmental Foundation (Deutsche Bundesstiftung Umwelt DBU), European Union and BMBF are gratefully acknowledged.

\section{Authors' contributions}

NW developed the method to fabricate micromirrors comprising the substructures for planarization and investigated the fabrication results. AT was a major contributor in technological support. NW was a major contributer in writing the manuscript. All authors read and approved the final manuscript.

\section{Funding}

This research was supported in part by the Doctoral Scholarship Program of the German Federal Environmental Foundation (Deutsche Bundesstiftung Umwelt DBU), European Union under grant ENER/FP7/284533/NEXT-BUILDINGS and the BMBF under grant 13N14517, which the authors gratefully thank.

\section{Availability of data and materials}

All data generated or analysed during this study are included in this published article. 


\section{Competing interests}

The authors declare that they have no competing interests.

\section{Author details}

${ }^{1}$ Institute of Nanostructure Technologies and Analytics (INA), Faculty of Electrical Engineering and Computer Science, Department of Technological Electronics, University of Kassel, Heinrich-Plett-Strasse 40, 34132 Kassel, Germany.

${ }^{2}$ Center for Interdisciplinary Nanostructure Science and Technology (CINSaT), Heinrich-Plett-Strasse 40, 34132 Kassel, Germany.

Received: 21 April 2020 Accepted: 1 November 2020

Published online: 09 November 2020

\section{References}

1. Ko WH (2007) Trends and frontiers of MEMS. Sens Actuators A 136(1):6267. https://doi.org/10.1016/j.sna.2007.02.001

2. Jha AR (2008) MEMS and nanotechnology-based sensors and devices for communications, medicals and aerospace applications, 1st edn. Taylor \& Francis, Boca Raton, pp 1-39

3. Jäkel A, Clobes J, Li Q, Viereck V, and Hillmer H (2010) Integration of actuable MEMS in networked sensing systems. INSS2010, Kassel, Germany, IEEE, 61-64. doi:https://doi.org/10.1109/INSS.2010.5573294.

4. Hillmer H, Schmid J, Stadler I (2003) Mikrospiegelarray. EU Patent 1700152 B1, 15 Dez 2003.

5. Hillmer H, Schmid J, Stadler I (2003) Micromirror array. US Patent 7677742 B2, 15 Dez 2003.

6. Pizzi M, Koniachkine V, De Martiis O, and Marbach R (2003) New type of micro-mechanical shutter arrays for replacement of infrared photodetector arrays. Optical MEMS 2003, Waikoloa, HI, USA, IEEE, 173-174. doi:10.1109/OMEMS.2003.1233521

7. Ohring M (2002) Materials science of thin films: deposition and structure, 2nd edn. Academic Press, San Diego, pp 742-754
8. Sinha AK Shen TT (1978) The temperature dependence of stresses in aluminum films on oxidized silicon substrates. Thin Solid Films 48(1):117126. https://doi.org/10.1016/0040-6090(78)90337-1

9. Hingerl K, and Cobet C (2014) Characterization of Al-micro mirrors: film properties/ origin of strain - EU Project MEM4WIN. Technical report, ZONA Johannes Kepler University Linz.

10. Abermann R (1990) Internal stress of vapour-deposited aluminium films: Effect of $\mathrm{O} 2$ and water vapour presented during film deposition. Thin Solid Films 186(2):233-240. https://doi.org/10.1016/0040-6090(90)90145 $-4$

11. Hillmer H, Al-Qargholi B, Kahn MM, Worapattrakul N, Wilke H, Woidt C, Tatzel A (2018) Optical MEMS-based micromirror arrays for active light steering in smart windows. Jpn J Appl Phys. https://doi.org/10.7567/ JJAP.57.08PA07

12. Iskhandar MSQ, Al-Qargholi B, Khan MM, Tatzel A, Luo H, Nazemroaya S, Li Q, Hillmer H (2019) Development of optical MEMS-based micromirror arrays for smart window applications: implementation of subfield addressing and reliability measurements. Yearbook surface technology, vol 52. Leuze Publishing House, Saulgau, pp 93-107

13. Tarraf A, Daleiden J, Irmer S, Prasai D, Hillmer H (2004) Stress investigation of PECVD dielectric layers for advanced optical MEMS. JMM 14(3):317323. https://doi.org/10.1088/0960-1317/14/3/001

14. Petersen KE (1982) Silicon as a mechanical material. Proc IEEE 70(5):420457. https://doi.org/10.1109/PROC.1982.12331

15. Guo LJ (2007) Nanoimprint Lithography: Methods and Material Requirements. Adv Mater 9(4):495-513. https://doi.org/10.1002/adma.200600882

16. Schift $H$ (2008) Nanoimprint lithography: an old story in modern times? A review. J Vac Sci Technol B 26(2):458-480. https://doi.org/10.1116/1.28909 72

\section{Publisher's Note}

Springer Nature remains neutral with regard to jurisdictional claims in published maps and institutional affiliations.

\section{Submit your manuscript to a SpringerOpen ${ }^{\circ}$ journal and benefit from:}

- Convenient online submission

- Rigorous peer review

- Open access: articles freely available online

- High visibility within the field

- Retaining the copyright to your article

Submit your next manuscript at springeropen.com 